\title{
Uso de extratos naturais como inibidores de corrosão para o aço AISI 304
}

\author{
Juliana Araujo da Silvaa*, Graziella Sarpe Capoa, Maurício Masaru de Souza Ribeiroa, Maurício \\ Marques Pinto da Silva ${ }^{a}$ \\ a Faculdade São Bernardo, São Bernardo do Campo, 09715-020, São Paulo, Brasil. \\ *julianaraujo33@gmail.com
}

Recebido: 6 agosto 2018 / Aceito: 22 setembro 2018 / Publicado online: 2 janeiro 2019

\begin{abstract}
Resumo
Essa pesquisa objetivou analisar a aplicação de extratos naturais na inibição de corrosão do aço AISI 304 em meio ácido. Os extratos aquosos e etanólicos das folhas desidratadas e moídas de Ilex paraguariensis A.St.-Hil e Camellia sinensis (L.) Kuntze, que foram obtidas na Zona Cerealista da cidade de São Paulo, foram previamente analisadas granulometricamente e submetidas à agitação magnética. A avaliação da proteção à corrosão foi feita com a imersão de placas de aço em alíquotas dos extratos aquosos e etanólicos durante 30 minutos. O potencial de corrosão (Ecorr) foi determinado utilizando eletrodo de calomelano saturado como referência. Na ausência de inibidores (ensaio preliminar), o Ecorr foi igual a - $158 \mathrm{mV} / \mathrm{ECS}$. O Ecorr do extrato aquosos de I. paraguariensis foi de - $138 \mathrm{mV} / \mathrm{ECS}$ e o de C. sinensis $-149 \mathrm{mV} / \mathrm{ECS}$. Os extratos etanólicos de I. paraguariensis e C. sinensis apresentaram Ecorr iguais a -58 e $-143 \mathrm{mV} / \mathrm{ECS}$, respectivamente. Apenas o extrato etanólico da Ilex paraguariensis apresentou resultado significativo na inibição do processo corrosivo no metal em estudo; desta forma, este apresenta potencial uso como inibidor de corrosão do aço AISI 304.
\end{abstract}

Palavras-chave: Anticorrosivos, Camellia sinensis, Ilex paraguariensis.

\section{Use of natural extracts as corrosion inhibitors for AISI 304 steel}

\begin{abstract}
This research is based on the application of natural extracts in the inhibition of corrosion of AISI 304 steel in acid solution. The aqueous and ethanolic extracts of the dehydrated and ground leaves of Ilex paraguariensis and Camellia sinensis, which were obtained in the Cereal Zone of the city of São Paulo, were previously analyzed granulometrically and submitted to magnetic stirring.The evaluation of the corrosion protection was done with the immersion of steel plates of aqueous and ethanolic extracts for 30 minutes. The corrosion potential (Ecorr) was determined using a saturated calomel electrode as reference. In the absence of inhibitors (preliminary test) the Ecorr was equal to $-158 \mathrm{mV} /$ ECS. However, in the presence of I. paraguariensis aqueous extract, the Ecorr was - $138 \mathrm{mV} / \mathrm{ECS}$ and $-149 \mathrm{mV} / \mathrm{ECS}$ for the aqueous extract of $C$. sinensis. The ethanolic extracts of $I$. paraguariensis and $C$. sinensis presented potential corrosion results of $-58 \mathrm{mV} / \mathrm{ECS}$ and $-143 \mathrm{mV} / \mathrm{ECS}$, respectively. Only the ethanolic extract of I. paraguariensis presented significative results in the inhibition of the corrosive process in the metal study,thus, the ethanolic extract of I. paraguariensis presents potential use as corrosion inhibitor for AISI 304.
\end{abstract}

Keywords: Anticorrosive, Camellia sinensis, Ilex paraguariensis.

\section{Introdução}

A corrosão é definida como um fenômeno natural comum em diversos tipos de metais, sendo o resultado de reações químicas ou eletroquímicas entre o metal e o ambiente, podendo estar relacionado à esforços mecânicos, causando a degradação e até a perda do metal. Para cada tipo de metal existem inibidores distintos, que devem ser selecionados anteriormente. Essa prática é bastante comum na indústria para reduzir os gastos com manutenção de elementos metálicos que sofrem com a corrosão (Távora, 2007).

Três métodos de inibição de corrosão são os mais comuns: o método eletroquímico, o de proteção anódica e catódica (revestimentos orgânicos) e a inibição da reação catódica ou anódica utilizando agentes que reagem com os produtos da corrosão (Costa, 2017). A maioria dos agentes inibidores utilizados em indústrias são compostos orgânicos (Rocha \& Gomes, 2017).

Os inibidores de corrosão químicos podem ser potencialmente poluidores, por este motivo, atuais estudos estão pesquisando a eficiência de extratos de plantas ou materiais biodegradáveis para realizar esta função, podendo levar à formação de filmes na superfície do metal, devido à presença de compostos como alcaloides, flavonoides, entre outros (Rocha \& Gomes, 2017).

Nesse contexto, diversos estudos têm sido feitos sobre o potencial de extratos naturais como inibidores verdes contra a 
corrosão de metais (Teixeira, Santos, Rezende \& D'Elia, 2015; Nabel, Salah \& Mona, 2013; Loto, Loto, Popoola, 2011). A substituição dos compostos orgânicos por outros de origem natural e renovável, como extratos naturais (inibidores verdes), podem contribuir para a diminuição da degradação ambiental.

A erva mate (I. paraguariensis) é uma espécie vegetal nativa das regiões subtropicais e temperadas da América do Sul, o extrato desta erva e de outras folhas pode ser utilizado como inibidor de corrosão para aço inoxidável em soluções ácidas (Santos et al., 2017).

$\mathrm{O}$ extrato de folhas de chás preto e verde ( $C$. sinensis) também tem sido pesquisada quanto à sua utilização na inibição da corrosão, por conter polifenóis monoméricos e catequinas que inibem a corrosão (Teixeira et al., 2015).

Essa pesquisa objetivou analisar a aplicação de extratos naturais na inibição de corrosão do aço AISI 304 em meio ácido.

\section{Material e Métodos}

Os inibidores verdes foram preparados obtendo-se extratos aquosos e etanólicos das folhas desidratadas e moídas com auxílio de almofariz e pistilo de I. paraguariensis e $C$. sinensis, que foram obtidas na Zona Cerealista da cidade de São Paulo.

Utilizaram-se $10 \mathrm{~g}$ do material previamente analisados granulometricamente com peneira de 65 Tyler com abertura de $212 \mu \mathrm{m}$, submetidos à agitação magnética (com $100 \mathrm{~mL}$ de água fervente por 60 minutos) e filtradas em papel filtro. Outra extração sob agitação foi realizada utilizando etanol (grau P.A.) como solvente de extração em temperatura ambiente.

As placas de aço inoxidável austenítico tipo ABNT 304 $(680 \times 120 \times 1 \mathrm{~mm})$ foram lixadas manualmente $(320,400 \mathrm{e}$ 600 mesh), até a remoção de resíduos de sujeira acumulados no local, lavadas com água destilada e secas em corrente de ar quente, conforme a norma ASTM-A967-05.

$A$ avaliação da proteção à corrosão foi por meio da imersão das placas em alíquotas dos inibidores, durante 30 minutos em meio natural, à temperatura ambiente. Em seguida, as placas foram expostas em meio corrosivo por um período de 60 minutos, com a imersão em solução de ácido

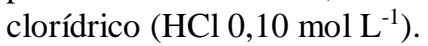

A variável resposta foi a diferença de potencial entre a placa e a solução (Potencial de circuito aberto - Eca), utilizando eletrodo de calomelano saturado (ECS) como referência na determinação do potencial de corrosão (Ecorr), sendo determinada no seguinte padrão: a cada minuto nos primeiros 10 minutos; a cada 2 minutos até completar 20 minutos e, a cada 5 minutos até o período de 60 minutos, tempo suficiente para determinação do valor de Ecorr. Os dados foram obtidos em duplicata. Um ensaio preliminar foi realizado, submetendo as placas diretamente ao meio corrosivo $(\mathrm{HCl})$ sem tratamento com os extratos naturais.

Para analisar os efeitos dos extratos de I. paraguariensis e $C$. sinensis como inibidores de corrosão, trataram-se estatisticamente os dados de Ecorr utilizando-se o teste $\mathrm{t}$ pareado.

\section{Resultados e Discussão}

As placas de aço AISI 304 na presença de inibidores apresentaram valores de Ecorr menos negativos, mais próximos de zero, em relação ao ensaio preliminar (Tabela 1).

Tabela 1. Valores médios de potencial de circuito aberto (Eca ( $\mathrm{mV} / \mathrm{ECS}$ ) obtidos na presença e ausência de extratos naturais (Et: etanólico e Aq: aquoso) e no ensaio preliminar $(\mathrm{HCl} \mathrm{0,10}$ $\left.\mathrm{mol} \cdot \mathrm{L}^{-1}\right)$ nas diferentes faixas de tempo analisadas.

\begin{tabular}{cccccc}
\hline \multirow{2}{*}{$\begin{array}{c}\text { Tempo } \\
\text { (min.) }\end{array}$} & $\mathrm{HCl}$ & \multicolumn{2}{c}{ Ilex paraguariensis } & \multicolumn{2}{c}{ Camellia sinensis } \\
\cline { 3 - 6 } & & $\mathrm{Et}$ & $\mathrm{Aq}$ & $\mathrm{Et}$ & $\mathrm{Aq}$ \\
\hline $0-10$ & $-199 \pm 19$ & $-44 \pm 4$ & $-171 \pm 4$ & $-191 \pm 23$ & $-194 \pm 17$ \\
$12-20$ & $-176 \pm 2$ & $-60 \pm 9$ & $-158 \pm 3$ & $-164 \pm 3$ & $-170 \pm 3$ \\
$25-40$ & $-169 \pm 3$ & $-68 \pm 3$ & $-148 \pm 4$ & $-154 \pm 4$ & $-160 \pm 3$ \\
$45-60$ & $-161 \pm 2$ & $-57 \pm 7$ & $-140 \pm 2$ & $-145 \pm 2$ & $-152 \pm 3$ \\
VE* $^{*}$ & $-158 \pm 1$ & $-58 \pm 4$ & $-138 \pm 7$ & $-143 \pm 3$ & $-149 \pm 2$ \\
\hline
\end{tabular}

* Valor de Ecorr. $\mathrm{O} \mathrm{HCl}$ foi utilizado na concentração $0,10 \mathrm{~mol} \mathrm{~L}^{-1}$.

O ensaio preliminar apresentou Ecorr médio igual a -158 $\mathrm{mV} / \mathrm{ECS}$ em um tempo de 60 minutos. Já em presença de extrato aquoso de I. paraguariensis o Ecorr foi de -138 e -149 $\mathrm{mV} / \mathrm{ECS}$ para o extrato aquoso de $C$. sinensis. Os extratos etanólicos de $I$. paraguariensis e $C$. sinensis, neste mesmo tempo, apresentaram valores de potencial de corrosão de -58 e $-143 \mathrm{mV} / \mathrm{ECS}$, respectivamente.

O valor menos negativo indica uma menor atividade corrosiva do aço, Carvalho (2015) também notou o comportamento de deslocamento do Eca para valores mais positivos para o aço carbono 1020 tratado com extrato natural.

As moléculas dos inibidores geram um filme protetor, adsorvendo na superfície metálica, o que leva à capacidade protetora de corrosão. Esta adsorção e eficiência estão relacionadas com a estrutura química e propriedades físicoquímicas do composto como, o grupo funcional, estrutura eletrônica da molécula, tamanho e comprimento da molécula, o tipo de ligação química e sua força, a capacidade de interligação, solubilidade ao meio, entre outros (Nabel et al., 2013).

Os compostos responsáveis pela ação antioxidante no $C$. sinensis e na I. paraguariensis, de acordo com Saito (2007), são os fenólicos, as folhas em base seca apresentam cerca de 10 a $20 \%$ destes compostos. Dentre outras características, os compostos fenólicos são responsáveis pela coloração das folhas e a capacidade de neutralizar radicais livres de oxigênio: estes compostos podem desempenhar atividades antioxidantes devido as suas características estruturais, como a afinidade com metais (Loto et al., 2011).

Esta afinidade se dá devido a ação como agente quelante dos compostos naturais, onde é realizada a ligação com o íon metálico em dois locais da molécula no grupo orto-difenólico dos dois aneis benzênicos de sua estrutura: este mecanismo da adsorção do filme protetor na superfície metálica pode ser representado por equações (Rocha, Gomes \& D'Ela, 2014 e Khan, Khaodhir \& Rotwiron, 2015).

Segundo as equações 1 e 2 , a molécula de inibidor que está presente na solução (Inibidor (sol)), substitui a molécula de água adsorvida na superfície metálica $\left(\mathrm{H}_{2} \mathrm{O}(\mathrm{ads})\right)$, não necessariamente obedecendo a proporção de 1:1. Para esta equação: (sol) significa molécula dissolvida na solução, (ads) moléculas adsorvidas na superfície, e " $\mathrm{x}$ " o número de 
moléculas de água que serão substituídas (Khan et al., 2015).

Depois da primeira etapa de substituição das moléculas de água adsorvidas, o inibidor pode se combinar com os íons metálicos $\left(\mathrm{M}^{2+}\right)$, que são gerados devido ao processo de oxidação, formando um complexo (Brandão, 2006).

$$
\begin{aligned}
& \text { Inibidor }_{(\mathrm{sol})}+\mathrm{xH}_{2} \mathrm{O} \rightarrow \text { Inibidor }_{(\mathrm{ads})}+\mathrm{H}_{2} \mathrm{O}_{(\mathrm{sol})} \\
& \mathrm{M}^{2+}+\text { Inibidor }_{(\mathrm{ads})} \rightarrow\left[\mathrm{M}-\text { Inibidor }_{(\mathrm{ads})}^{+2}\right.
\end{aligned}
$$

Este mecanismo é ilustrado graficamente (Figura 1) onde, foi possível observar a tendência de comportamento da placa metálica em estudo, quando atacada pelo meio corrosivo em presença e em ausência dos extratos naturais. A curva de Eca para o extrato etanólico de I. paraguariensis foi a única, que mostrou comportamento inibitório de corrosão, as curvas de Eca dos demais extratos foram semelhantes ao ensaio preliminar, sendo assim, não apresentaram efeito inibitório.

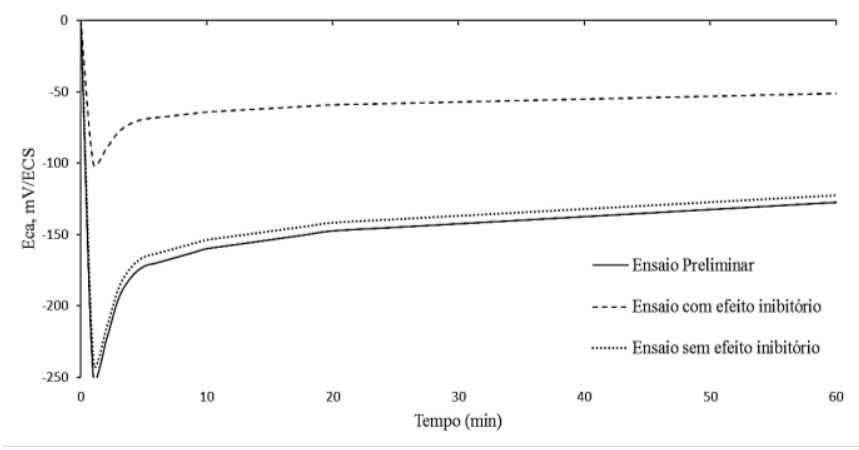

Figura 1. Comparação dos valores de Eca no efeito inibitório de extratos naturais na corrosão do aço AISI 304 em meio de $\mathrm{HCl}$.

No contato inicial do metal com o meio corrosivo, a tendência do valor de Eca é subir abruptamente devido à busca do equilíbrio, nos 10 primeiros minutos do ensaio preliminar, os valores de Eca variaram de forma não linear. Este comportamento pode indicar o ataque inicial da solução de ácido clorídrico à placa de aço já que, segundo Tussolini, Spagnol, Gomes, Cunha \& Rodrigues (2007), o aço tipo ABNT 304 não resiste a ácidos como o clorídrico diluído, sendo necessária a adição de inibidores de corrosão para que o mesmo não degrade.

Na placa tratada com inibidores naturais, nos mesmos dez primeiros minutos, os valores de Eca se apresentaram menos negativos do que o ensaio preliminar. Porém, ocorreu a oxidação do metal, indicado por valores de Eca mais negativos, conforme liberaram-se os íons provenientes da oxidação, estes reagiram com a molécula do inibidor formando o complexo que foi responsável pela eficiência inibitória da corrosão (valor de Ecorr estabilizado).

Assim, pode-se inferir que o efeito de adsorção das moléculas orgânicas (compostos polifenóis ou heterocíclicos) presentes no extrato de $C$. sinensis e $I$. paraguariensis provavelmente contribuíram na preservação do aço, com a formação de uma película protetora (Rocha \& Gomes, 2017)

Segundo Singh, Ahamad \& Singh (2011), em seu estudo do efeito inibitório de corrosão por compostos naturais, a eficiência na redução no potencial de corrosão na superfície metálica de aço carbono atacada por ácido clorídrico, também foi atribuída a formação de um filme protetor do extrato de Pongamia pinnata.

Então, pôde-se testar que as diferenças (analisadas pelo teste-t pareado) entre o ensaio preliminar e com inibidores etanoicos são diferentes de zero, em que o extrato de $I$. paraguariensis apresentou valor $86 \%$ menor e o extrato de $C$. sinensis $7 \%$, ou seja, a proteção contra a corrosão se mostrou mais eficiente em soluções etanólicas, para o extrato de $I$. paraguariensis devido à ação polar e apolar do etanol, para o extrato de $C$. sinensis o efeito inibitório não se mostrou tão significativo.

Inicialmente a parte apolar do solvente removeu óleos, graxas, pigmentos e esteróis, que podem estar presentes nos extratos naturais, o que facilitou a extração dos compostos de interesse, onde a parte polar extraiu as substâncias polifenólicas, como os flavonoides. As soluções aquosas de ambos os extratos naturais em estudo, teriam melhor eficácia, se antes fosse realizada uma etapa de extração de impurezas (moléculas não importantes para o presente estudo) através de um solvente apolar (Rocha et al., 2014).

\section{Conclusões}

Dentre os extratos analisados, o extrato etanólico de $I$. paraguariensis., apresentou valor significativamente melhor que o ensaio preliminar, para a amenização do efeito corrosivo sobre a placa do aço AISI 304 nas condições experimentais avaliadas.

Para validação do potencial uso de $C$. sinensis e $I$. paraguariensis como inibidores de corrosão para o aço AISI 304 , deve-se quantificar a concentração de polifenóis nos extratos das ervas nos diferentes métodos de extração, além de realizar testes com outros meios corrosivos, também alterando o tempo de imersão da placa nos extratos naturais.

\section{Referências}

American Society for Testing and Materials. (2005). Standard Specification for Chemical Passivation Treatments for Stainless Steel Parts (A967-05). Pennsylvania: ASTM International. doi: 10.1520/A0967-05

Carvalho, R. H. R. (2015). Extração supercrítica de óleo de Sesamum indicum L. e aplicação como inibidor de corrosão em aço AISI 1020 através de sistemas microemulsionados (Tese de doutorado). Universidade Federal do Rio Grande do Norte, Natal, Rio Grande do Norte, Brasil. Recuperado de https://repositorio.ufrn.br/jspui/handle/123456789/22444

Costa, S. N. (2017). Compostos derivados do Imidazol como inibidores da corrosão do aço carbono em meio ácido (Dissertação de mestrado). Universidade Federal do Ceará, Fortaleza, Brasil. Recuperado de http://www.repositorio.ufc.br/handle/riufc/22805

Khan, G., Kazi, S. N., Basirun, W., Binti, H., Ali, M., Faraj, F. \& Mustafa Khan, G. (2015). Application of Natural Product Extracts as Green Corrosion Inhibitors for Metals and Alloys in Acid Pickling Processes-A review. International Journal of Electrochemical Science, 10(8), 61206134.

Loto, C.A., Loto, R.T. \&Popoola A.P.I (2011). Inhibition Effect of Extracts of Carica Papaya and Camellia Sinensis Leaves on the Corrosion of Duplex $(\alpha \beta)$ Brass in $1 \mathrm{M}$ Nitric acid. International Journal of Electrochemical Science, 6(10), 4900-4914.

Nabel, A. N., M. A., Yousef, M. A. \& Tawfik, S. M. (2013). Impact of Synthesized and Natural Compounds in Corrosion Inhibition of Carbon Steel and Aluminium in Acidic Media. Recent Patents on Corrosion Science (Discontinued), $\quad 3(1), \quad 58$ 68. doi: 10.2174/2210683911303010007

Rocha, J. C. \& Gomes, J. A. C. P. 2017. Inibidores de corrosão naturais - 
Proposta de obtenção de produtos ecológicos de baixo custo a partir de resíduos industriais. Revista Matéria (Rio de Janeiro), 22(suppl.1), e11927. doi: 10.1590/s1517-707620170005.0263

Rocha, J. C., Gomes, J. A. C. P. \& D'Elia, E. (2014). Aqueous extracts of mango and orange peel as green inhibitors for carbon steel in hydrochloric acid solution. Materials Research, 17(6), 1581-1587. doi: 10.1590/1516-1439.285014

Santos, A. M., Almeida, T. F., Cotting, F., Aoki, I. V., Melo, H. C. \& Capelossi, V. R. (2017). Evaluation of Castor Bark Powder as a Corrosion Inhibitor for Carbon Steel in Acidic Media. Materials Research, 20(Suppl. 2), 492-505. doi: 10.1590/1980-5373-mr-20160963

Singh, A., Ahamad, I. \& Singh, V. K. (2011). Inhibition effect of environmentally benign Karanj (Pongamia pinnata) seed extract on corrosion of mild steel in hydrochloric acid solution. Journal Solid State Electrochem.2011. 15: 1087. doi: 10.1007/s 10008-010-1172-z

Távora, M. P. (2007). Avaliação do efeito de particulados sólidos na eficiência de um inibidor de corrosão recomendado para meios salinos com $\mathrm{CO}_{2}$ (Dissertação de mestrado). Universidade Federal do Rio Grande do Norte, Natal, Rio Grande do Norte, Brasil. Recuperado de https://repositorio.ufrn.br/jspui/handle/123456789/12858

Teixeira, V. M., Santos, E. C., Rezende, M. J. C. \& D’Elia, E. (2015). Estudo da ação Inibidora do Extrato de Camellia sinensis na Corrosão do AçoCarbono 1020 em HCl $1 \mathrm{~mol} \mathrm{~L}^{-1}$. Revista Virtual de Química, 7(5), 17801794. doi: 10.5935/1984-6835.20150102

Tussolini, M., Cristiane, S., Gomes, E. C., Cunha, M. T. \& Rodrigues, P. G. P. (2007). Estudo do comportamento eletroquímico do benzotriazol e benzimidazol na oxidação do aço inoxidável tipo ABNT 304. Revista Escola de Minas, 60(1), 41-44. doi: 10.1590/S037044672007000100006

\section{Licença Creative Commons CC BY 4.0}

Este artigo foi publicado com acesso aberto para distribuíção sob os termos do Licença de Atribuição Creative Commons, que permite uso irrestrito, distribuição, e reprodução em qualquer meio, desde que o trabalho original seja devidamente citado. 